Keh-Ming Lin, M.D., M.P.H.

Freda Cheung, Ph.D.

One of fastest-growing population groups in recent decades, Asian Americans represent a vastly diversified and rich mixture of cultures, languages, beliefs, and practices, many of which differ widely from those of E uropean Americans. As immigrants, Asian Americans have experienced and continue to experience various emotional and behavioral problems. However, they tend to underuse existing services except those that are culturally appropriate and linguistically compatible. Misdiagnosis frequently occurs, and the existence of culture-bound syndromes points to a lack of precise correspondence between indigenous labels and established diagnostic categories. D ue to Asian traditions of viewing the body and mind as unitary rather than dualistic, patients tend to focus more on physical discomforts than emotional symptoms, leading to an overrepresentation of somatic complaints. Traditional practices and healing methods are frequently used to alleviate distress both before and after patients and their family members approach the conventional mental health care system. H elp seeking typically is a family venture. Asian patients respond well to highly structured therapeutic interventions such as those used in behavioral, cognitive, and interpersonal models. When applying pharmacotherapy, clinicians should pay attention to Asians' unique responses to psychotropics, especially in regard to dosage requirements and side effects. Research in this area as well as on other important issues is in the early stage of development. (Psychiatric Services 50:774- 780, 1999)

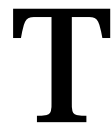
he growth of the Asian-American population in recent years has been phenomenal. Since 1960 it has increased by more than 100 percent every decade and has expanded from less than 1 million to more than 8.5 million in three decades, representing 3.3 percent of the total U.S. population (1). In all likelihood, this trend will continue into the next century. Thus clinicians can ill afford to remain unfamiliar with issues that are of particular importance in providing care to this population.

Furthermore, as the technology of communication continues to advance, various aspects of mental health practices will likely become increasingly global and cross-cultural. As Asians represent more than 50 percent of the earth's population, their perspectives on mental health care warrant more careful attention (2). Understanding the mental health issues that confront Asian Americans takes on a special significance, because they tend to retain the cultural traits from their ancestral land. Furthermore, many of the issues facing Asian Americans may also be relevant for Asians outside of $\mathrm{N}$ orth America.

Like terms used for other major
The authors are affiliated with the department of psychiatry and the Research Center on the Psychobiology of Ethnicity at H arbor-University of California at L os Angeles M edical Center, 1124 W est Carson Street, B-4 South, Torrance, California 90502 (e-mail, linkeh@harbor2.humc.edu). population groups, such as $\mathrm{H}$ ispanic, $\mathrm{N}$ ative American, African American, and Caucasian, the terms Asian and Asian American may convey a false sense of homogeneity. In reality, when examined closely, each of these commonly used labels refers to a group that encompasses tremendous heterogeneity. Asian Americans, for example, represent more than 28 Asian groups (3), each with its distinct culture, language, religious traditions, dietary practices, and immigration history. Furthermore, each cultural groupsuch as Chinese, Japanese or $\mathrm{Khmer-includes}$ wide variations in educational levels, family income, residential preference, exposure to war trauma, and levels of acculturation. Such intragroup diversity calls into question the validity and utility of any attempt at generalization and points to the danger of stereotyping.

H owever, at the same time, most of these cultural traditions, though distinct, have been in constant interaction and exchange for several millennia and reflect repeated large-scale migrations and intermixture of populations (4). $M$ ajor traditions and belief systems, such as Buddhism and Confucianism, permeated a large part of the Asian continent and continue to exert profound influences on the general populace $(5,6)$. They helped shape world views and value systems that often are different from and at times clearly at odds with those found in E uropeanAmerican traditions.

Partly because of such influences, Asians and "Westerners" diverge significantly in conceptualizations of the self, the relationship between the self and social groups, and the relationship between the body and the mind (7-9). Such differences are likely to have profound and pervasive influences on the 
recognition and reporting of psychiatric symptoms, the process of help seeking, the use of mental health care systems, and the response to various contemporary mental health treatment modalities. In addition, emerging data indicate that, for both genetic and environmental reasons, Asians who are treated with psychotropic medications may differ from Caucasians in their dosage requirements and side effect profiles (10).

This paper reviews mental health issues for Asian Americans in the areas of utilization of mental health services, psychiatric diagnosis, cultural patterns of illness presentation, the role of indigenous treatments, the role of the family in treatment, and use of psychosocial and pharmacotherapeutic interventions.

\section{Utilization of}

\section{mental health services}

Extremely low admission rates to state hospitals have been consistently documented for Asians in the past (11). In recent years, these findings have been augmented by equally substantive reports of their low utilization of outpatient mental health services (12-14).

E arlier researchers suggested that the low utilization rates might reflect low rates of psychopathology among Asians. This line of reasoning considered Asians a "model minority group," who, despite discrimination and the adversities generally associated with being immigrants, remained resilient and immune from emotional breakdown and behavioral problems. However, when Asian patients were more closely examined, their conditions were significantly more severe and chronic than those of patients of other cultural backgrounds and consequently required more intensive treatment and longer hospitalization or outpatient care.

Taken together, these data suggested that Asians rarely utilized services because they often did not reach the service systems until very late in their help-seeking process (15), when their condition was extremely severe and all other resources had been exhausted (16). This hypothesis was indeed supported in other well-designed studies (17). The data showed that Asian patients with schizophrenia typically
E ditor's Note: This paper is the second in an occasional series on the mental health needs of nonwhite populations in the United States. The aim of the series is to improve awareness of the variety of psychiatric needs in the increasingly diverse population of the U.S. The first paper in the series- on African Americans-was published in $M$ arch. F uture papers will address the psychiatric needs of $\mathrm{N}$ ative Americans and Latinos. Carl C. Bell, M.D., chief executive officer of the $\mathrm{Com}$ munity $\mathrm{M}$ ental $\mathrm{H}$ ealth Council, I nc., in Chicago, is the editor of the series.

were contained within the family and the community and generally did not reach the mental health system until three years after the initial onset of their psychotic symptoms. This delay was significantly longer than for their counterparts in Caucasian and African-American groups, who entered the mental health system an average of one and one and a half years, respectively, after onset of symptoms.

Based on these findings, specialized mental health programs for Asian Americans have been successfully established in many parts of the country $(18,19)$, including Boston, L os Angeles, N ew York, Portland, San F rancis$\mathrm{CO}$, and Seattle. Staffed with bilingual and bicultural professionals and paraprofessionals who together cover a wide range of Asian languages and cultures, these clinics strive to provide culturally appropriate services in locations close to major Asian communities. They have been strikingly successful, at least in terms of increasing utilization and shortening the delay between onset of symptoms and contact with the mental health system.

Almost invariably, these clinics were started amid intense political struggles, with funding agencies frequently expressing doubts about their ability to survive, because of the pervasive belief that the need and demand for them did not exist. H owever, once situated in the community, they were filled to capacity within a short time. $M$ any of these clinics expanded into full-fledged community mental health centers within a relatively short period.

Although these specialized programs have made a visible impact on the mental health care of Asian Americans, service barriers to the overall mental health system and low utilization of services continue to be major issues, both in the public and the private sectors $(20,21)$.

\section{Psychiatric diagnosis}

Besides the availability and accessibility of services, another major issue that deserves closer scrutiny is the degree of fit between current psychiatric diagnostic systems and assessment practices on the one hand and AsianAmerican subcultures on the other. Current psychiatric theories about etiology and nosology largely derived from generations of clinicians' work with European and North American patients (22-24). M ost research testing the coherence and relevance of these theories has also been conducted with Caucasian subjects. Consequently, it is unclear to what extent such a nosologic system might be useful for patients with other cultural and ethnic backgrounds.

What is clear, however, is that misdiagnosis often occurs in cross-cultural situations (25-27) and that assessment tools developed in one cultural setting may be found invalid when translated (28). We recently examined the relationship between ethnicity and psychiatric diagnosis among patients treated in a major urban mental health system and found that atypical diagnoses were significantly overrepresented in all ethnic minority groups, including Asian Americans. (Yeh M, Chung R, Lin K, unpublished data, 1997). These results further support the hypothesis that there is greater ambiguity and difficulty when applying the DSM diagnostic system with non-Caucasian patients.

\section{Cultural patterns of illness}

Somatization

One of the ongoing debates about the relationship between culture and psychopathology has to do with the long- 
reported tendency of Asians to present primarily with somatic symptoms when in distress $(29,30)$. The tendency of Asian psychiatric patients to focus on physical discomfort while ignoring or suppressing the reporting of emotional symptoms has been a challenge for clinicians who work with this population.

Various theories have been proposed for such a phenomenon, including speculations about Asians' relative lack of psychological mindedness and a putative deficiency of psychological descriptive terms in Asian languages. Both have since been refuted as unfounded. A newer generation of researchers, represented by Kleinman (31) and his colleagues, showed that for the majority of the Asian patients, somatic symptoms are used as culturally sanctioned "idioms of distress," whose mechanism may thus be quite distinct from the process of somatization as commonly understood.

A series of studies conducted by Cheung $(32,33)$ in $\mathrm{H}$ ong $\mathrm{K}$ ong further supported this explanation. She found that although patients typically focused on their somatic complaints when visiting a physician, they were often fully aware of their emotional problems as well as stresses derived from social relationships that might be related to their emotional as well as somatic symptoms. Based on these findings, she suggested that most patients selectively presented their symptoms according to what they perceived as appropriate and tended to focus only on somatic suffering while in health care settings.

Similarly, in a longitudinal follow-up study of Vietnamese refugees (34), most of those who were interviewed initially reported only physical symptoms (35). H owever, their response to the Cornell M edical Index showed extremely high mean psychological as well as somatic scores, indicating that when they were asked specifically, they did not have difficulty reporting psychological symptoms (36). Other studies using a variety of self-report instruments have al so supported the thesis that Asians have no difficulties in identifying and reporting psychological symptoms $(27,32)$.

One fundamental issue that might be at the crux of the matter is the rela- tionship between the body and the mind. The Western view that emphasizes the separateness of the two has roots in the Greek philosophies and has been firmly established since the time of Descartes. This dichotomous model not only has provided the foundation of modern medicine, but also has exerted profound influences on practically every aspect of modern Western life, including how distress is expressed (37-39).

In diametric opposition to this perspective, Asian traditions have always regarded body and mind as one (40). Despite Western influences, most contemporary Asians continue to adhere to such a unitary model, which consciously or unconsciously permeates all aspects of their lives, including language and help-seeking preferences. Therefore, Asians, who are less inclined to dichotomize body and mind, tend to report distress accordingly. This tendency, added to a natural tendency to be reticent about emotions that may be linked to a person's private life, may result in the exclusive reporting of somatic symptoms.

Although the meaning and nature of somatization among Asians are awaiting further clarification, what is clear is that a somatic presentation of symptoms often predominates during an Asian patient's first mental health contact (37). It is also clear that for the majority of these patients, psychological and behavioral symptoms will emerge if the clinician inquires specifically about these symptoms. In view of the belief in the unity of body and mind, it may also be crucial for clinicians not to focus predominantly on the psychological side of the patient's suffering, but to present a formulation compatible with the patient's cultural orientations.

\section{Culture-bound syndromes}

The discrepancy between $C$ hinese and American psychiatrists in the use of "neurasthenia" ("shenjing shuairuo" in $M$ andarin Chinese) as a diagnostic term has been the subject of a major international debate for the last two decades. N eurasthenia was popular as a disease concept in North America and $E$ urope during the late 19th and early 20th century, but it gradually lost its currency and is now an obsolete concept among contemporary Western physicians. Paradoxically, after the concept was transplanted to $\mathrm{China}$ and other parts of Asia around the turn of the century, it quickly took root and flourished $(41,42)$.

The ambiguity of its meaning in the past apparently has encouraged its inappropriate use, to the extent that some surveys showed up to a 10 percent prevalence rate in the general population in China (43). In addition, there is documented evidence in Japan and China that physicians collude with patients with schizophrenia and their families in using the term neurasthenia to minimize stigma (44).

Such reports led to serious doubts about the validity of the concept of neurasthenia as used in China. In 1982 Kleinman (45) reported that 87 percent of patients diagnosed by Chinese psychiatrists as having neurasthenia could be reclassified as having major depression according to DSM-III criteria. These findings triggered extensive debates and eventually led to operationalization of a more narrowly defined diagnosis of neurasthenia by Chinese psychiatrists.

Using such operationalized criteria sets as well as one subsequently developed by the World Health Organization's International Classification of Diseases, Version 10 (ICD-10) (46), recent studies demonstrated that the illnesses of a substantial number of patients, both in clinics and in community settings, fit best with a diagnosis of neurasthenia rather than with any of the DSM categories. F or example, an epidemiological study of Chinese Americans in Los Angeles found that 6.7 percent of respondents experienced ICD -10 neurasthenia, and less than 50 percent of them had aDSM - III-R diagnosis (47). Our clinical study involving 486 subjects from L os Angeles, China, Hong Kong, and Taiwan showed similar results ( $L$ in $K M$, Weiss $M$, Zheng, UP, unpublished data, 1995).

Korean and Korean American patients suffering from similar mixtures of a wide variety of somatic and emotional symptoms often label themselves as suffering from "hwa-byung," which literally means both "fire disease" and "anger disease" (48). As these terms suggest, patients believe that their problems are caused by 
chronic unresolved anger that led to the imbalance of the body by the excessive accumulation of the fire element, as conceptualized in Oriental medicine theories. A community survey in Los Angeles showed that 12 percent of K orean Americans residing in the city labeled themselves as suffering from hwa-byung and that these subjects had a significantly elevated risk for also suffering from DSM -III-R major depression (49). The findings were comparable to those of studies conducted in Korea, using similar research strategies (50).

$\mathrm{H}$ wa-byung is another example of how Asians conceptualize the bodymind relationship. Although the concept denotes the existence of chronic social stress as well as emotional responses, most patients with hwabyung believe that their problems are primarily physical. Some are even convinced that the anger and the chronic imbalance have resulted in the accumulation of harmful substances in the epigastrial area in the form of a mass that could kill them if untreated.

D ue to a lack of precise correspondence between these indigenous labels and DSM diagnostic categories, both hwa-byung and shengjing shuairuo (neurasthenia) were placed in appendix I of DSM -IV (51), "O utline for Cultural Formulation and Glossary of Culture-Bound Syndromes," as examples of culturebound syndromes. Although controversy about the wisdom of this decision and the meaning of culturebound syndrome continues, such inclusions do highlight the importance of cultural factors in shaping symptom manifestation and clustering. $M$ any of the so-called culture-bound syndromes appear to apply to cases with features of both mood disorders and somatoform disorders, suggesting that for non-Western populations the boundary for these two domains may not be as distinct as DSM proposes (52). Research on these syndromes may inform future development in psychiatric nosology.

\section{Indigenous or alternative treatment}

As shown in recent studies, alternative or unconventional treatments are widely available and are frequently used by patients with all kinds of afflictions, including those with primarily psychiatric problems $(53,54)$. Nationwide, the annual out-of-pocket expenditure on alternative care, including meditation, herbal medicine, and spiritual healing, has been estimated to surpass the total outpatient medical cost (55).

The literature strongly suggests that such treatment and healing methods may be even more widely used by patients with ethnic minority backgrounds (56). In practically all contemporary Asian societies, multiple indigenous care and healing traditions continue to exist and thrive side by side with the conventional Western health care system. Traditional practitioners, who include not only Oriental medicine doctors and acupuncturists but also various kinds of herbalists, bone setters, psychic healers, and spiritual mediums, also are widely available in Asian-American communities (57).

Asian-American psychiatric patients often consult these practitioners before contacting the mental health system and continue to utilize their services even after entering psychiatric care. Because patients understandably do not volunteer the information that they are using alternative treatments, mental health clinicians generally are unaware of the extent of these contacts, their effects on patients' conditions, and their possible contributions to premature termination of therapy. Also remaining largely unclarified is the extent to which these traditional practices might interact with modern mental health care. E merging data now strongly suggest that some of the commonly used herbs might interact with psychotropic medications through pharmacokinetic or pharmacodynamic mechanisms (56).

Aside from whether and to what extent any of these traditional practices and healing methods might be efficacious in ameliorating psychiatric symptoms or what their potential for toxic effect might be, it is essential that clinicians working with Asian Americans be familiar with the alternative practices that are widely available and frequently used by them, as well as the health beliefs behind these practices.
Such understanding and efforts at accommodation would enhance trust and therapeutic alliance and thereby provide clinicians with the opportunity to intervene when necessary (58).

\section{The role of the family}

Reflecting the deeply ingrained individualistic orientation in Western cultures, contemporary American mental health practice focuses its attention primarily on the patient as an individual and to a large extent neglects the fact that a person is always a member of a social group. The involvement of family members with the patients and their treatment is often regarded by mental health professionals as intrusive and pathological (59). Concerns about confidentiality often further limit clinicians' contacts with the family. D espite the emergence in recent years of research findings showing the importance of involving family members in the care of psychiatric patients and despite the concerted efforts of family groups such as the Alliance for the M entally III in promoting collaboration between family members and professionals, this individualistic emphasis remains strong.

The exclusion of the family poses an even larger problem for Asian patients. In contrast to the W estern emphasis on the individual, Asian traditions in general regard the family as the basic unit of the society. A family member'sillness is considered a threat to the homeostasis of the family, which often leads to the mobilization of the family's resources. H elp seeking typically is a joint family venture rather than a personal decision.

An Asian patient's first mental health visit often is initiated by a family member and not infrequently is preceded by phone calls from one or more family members. In a cross-cultural study comparing treatment responses of Asian and Caucasian patients with schizophrenia, the majority of the Asian patients were accompanied by one or more family members (60). U nless specifically instructed, family members typically assume that it is their responsibility to be with the patient when the patient talks to the clinician. Clinicians unfamiliar with the cultural underpinning of such behavior might regard it as a 
manifestation of the patient's pathological dependency or the family's overinvolvement. Attempts at confronting such "inappropriate" behavior may lead to confusion and a sense of humiliation and may result in premature termination.

\section{Psychosocial interventions and psychotherapy}

Contrary to earlier impressions, Asian patients are often receptive of and are likely to benefit from psychotherapeutic interventions (61-63). I mportant ingredients of successful therapy, such as the instillation of hope, the fostering of trust, the identification or construction of "explanatory models" that make sense to the patient, and the mobilization of forces toward recovery (64), likely apply to patients irrespective of cultural backgrounds.

However, because the format and the context of therapeutic and healing systems are drastically different from culture to culture, it is important that an orientation phase, during which the purpose and ground rules of therapy are specifically discussed, be offered before the inception of psychotherapy. The therapist should make a conscientious effort to identify with roles familiar to the patient, such as a medical professional or a teacher. Teachers in traditional Asian societies not only are regarded as instructors of technical knowledge but also are idealized as moral exemplars and counselors. Strict neutrality, as promoted by orthodox psychoanalysis, may be perceived as an indication of the therapist's indifference or rejection (65). To demonstrate efficacy and gain credibility, therapists may have to take a more active therapeutic stance, such as by providing structure, giving advice, and acting as advocate when necessary and appropriate (66).

Although empirical data about the relative efficacy and utility of various forms of psychotherapy with Asian patients are limited, there are reasons to believe that the newer, highly structured therapeutic models, such as behavioral, cognitive, and interpersonal therapies, may work particularly well. Rationales behind behavioral interventions, such as progressive relaxation, systematic desensitization, and behavioral modification, are specific and relatively easy to grasp. Their results are demonstrable and measurable. Principles behind cognitive therapy often have echoes in traditional Asian beliefs. The emphasis of interpersonal therapy on roles and social relations also resonates well with Asian traditions. H owever, the effectiveness and utility of these specific methods with Asian-American patients should be systematically tested.

Recent studies have shown the importance of psychosocial rehabilitation in the care of patients with chronic mental illness (67). H owever, how these models would fit well in AsianAmerican cultural settings remains to be determined. Researchers need to involve Asian patients in their studies in this area.

\section{Pharmacotherapy}

Although many mental health practitioners were unaware of the findings until recently, a large number of reports-starting in the late 1950s, when psychotropics were first introduced-strongly suggest that Asian Americans may have a unique response pattern to these potent medications, especially in dosage requirement and side effect profiles (10). $D$ ata that have emerged in the last two decades have identified pharmacogenetic, pharmacokinetic, and pharmacodynamic mechanisms that are responsible for the differences in clinical responses that were reported earlier (68).

The literature indicates that for various reasons, Asian patients often respond to substantially lower doses of psychotropics than those recommended in standard textbooks and publications based on studies typically involving Caucasian subjects. Among the factors that might be responsible for this phenomenon, the most extensively studied are related to pharmacogenetics, especially findings on the structure and function of a group of drug-metabolizing enzymes called cytochrome P-450 isozymes. They play a crucial role in the metabolism of most psychotropic medications as well as other medications.

The activity of a number of these isozymes has been demonstrated to be significantly slower among Asians, including Chinese, Japanese, and Ko- reans, than among Caucasians. Some of these effects appear to be caused by specific mutations, such as the activity of CYP2D 6 and CYP2C 19, while the others appear to be due to environmental influences such as diet, notably the activity of CYP3A4 and CYP1A2. F or example, approximately one-third of Asians are homozygote for a mutation called CYP2D 6) (recently renamed CYP2D 6*10) that significantly slows down the activity of the enzyme CYP2D 6, which plays a crucial role in the metabolism of most traditional neuroleptics and tricyclic antidepressants (69). Asians also possess a specific mutation of CYP2C 19 ( $\mathrm{m} 2$ ) that is rarely seen in other ethnic groups. This mutation makes 20 percent of Asians poor metabolizers of drugs such as diazepam, compared with 3 percent of $\mathrm{C}$ aucasians.

Research data also suggest that the activity of other P-450 isozymes is slower in Asians even though no active mutations have been observed. Since these enzymes are highly responsive to environmental influences, crosscultural differences in lifestyles, including dietary practices, are most likely responsible for such lower enzyme activity. For example, CYP1A2 is inducible by char-broiled beef, cruciferous vegetables, and a high-protein diet, and CYP3A4 is inhibited by certain citrus fruits such as grapefruits and plant products such as corn. Because these enzymes are involved in the metabolism of some of the newer neuroleptics and antidepressants, it is reasonable to expect that Asians and Asian Americans would also be more sensitive to these medications as well.

Besides ethnic differences in drug metabolism, evidence also suggests that ethnic differences in pharmacodynamics - the pharmacological effects on the target organs-also exist. In psychiatry the most prominent example is the difference between Asians and Caucasians in the range of serum lithium concentrations regarded as therapeutic (68). In various parts of Asia, including Japan, China, $\mathrm{H}$ ong Kong, and Taiwan, optimal treatment with lithium was achieved with serum lithium concentrations below .8 $\mathrm{mE} \mathrm{q} / \mathrm{mL}$, in contrast to the range of .8 to $1.2 \mathrm{mE} \mathrm{q} / \mathrm{mL}$ generally regarded as therapeutic in Western countries. 
Such a substantial and consistent trans-Pacific difference suggests ethnic differences in the responsitivity of brain receptors to lithium.

Although the mechanisms responsible for ethnic differences in effects of psychotropics have not been completely clarified, the literature clearly indicates that for similar clinical conditions, Asian patients typically require lower doses of the same medication than Caucasian patients. In addition, Asian patients may experience side effects with greater severity or at a higher rate if treated with doses commonly used for their Caucasian counterparts.

Thus when starting an Asian-American patient on a new psychotropic medication, it is generally a good idea to begin with a dose approximately half the dosage usually recommended for $\mathrm{C}$ aucasians. $\mathrm{H}$ owever, this suggestion should not lead to rigid or stereotypical practice. In considering any generalization involving cross-cultural or cross-ethnic comparisons, one should be aware that substantial variability exists within any population (70). Although the majority of AsianAmerican patients respond to psychotropic doses lower than those generally prescribed for Caucasians, some may need doses similar to or even higher than those for "typical" Caucasian patients.

Also, although genetically based differences in drug metabolism most likely would remain stable over time, differences secondary to dietary and other lifestyle factors might alter dramatically as societies evolve. Thus these differences would need to be reassessed from time to time to ensure that earlier observations remain applicable to a particular population.

\section{Discussions and conclusions}

As is true for any cultural or ethnic group, issues that are relevant to the mental health of Asian Americans are multidimensional and complex. In this paper we selectively reviewed research findings that are most immediately relevant for clinicians providing care for patients with such ethnic or cultural backgrounds.

$M$ any other issues that are also crucial for an adequate understanding of the behavior of Asians and Asian
Americans, both normal and abnormal, have not been included in this brief survey because of the limitation of space and the need for a clinical focus. These issues include the history of different waves and modes of immigration, the effect of racism and discrimination, the history of trauma and refugee experiences, the impact of language on psychiatric assessment and treatment, the cultural appropriateness of assessment instruments, identity conflicts and resolution, and access to care related to health insurance coverage, to name a few. Although previous reviews have provided excellent coverage of some of these issues $(5,12)$, they will continue to evolve and confront researchers and clinicians. With the rapid changes in the social milieu and services systems in the U.S., including the emergence and permutation of managed care systems, we will constantly face new challenges in our search for optimal ways to provide care for this segment of the American population.

$\mathrm{H}$ igh-quality theory-based research is essential for addressing issues reviewed in this paper, as well as those listed above. Such research not only is essential for enhancing mental health professionals' ability to provide appropriate and effective care for the rapidly expanding Asian-American populations, but it will also contribute to providing better mental health care for more than half of the world's population residing in the Asian continent. At the same time it also will enrich the mental health field and help its insights and practices to become increasingly universal and globally applicable.

\section{Acknowledgment}

This work was partly supported by grant $\mathrm{M} \mathrm{H}-47193$ from the $\mathrm{N}$ ational Institute of $M$ ental $\mathrm{H}$ ealth.

\section{References}

1. US Bureau of the Census: Population Projections of the United States, by Age, Sex, Race, and Hispanic Origin: 1992-2050. Washington, DC, US Government Printing Office, 1992

2. D esjarlais R, E isenberg $L, G$ ood $B$ J, et al: World M ental $\mathrm{H}$ ealth: Problems and Priorities in Low-Income Countries. N ew York, Oxford U niversity Press, 1995

3. Asian and Pacific Islander American Popu- lation Statistics. M onograph series no 1. San Francisco, Asian American Health Forum, 1990

4. F airbank JK, Reischauer E O, Craig AM: East Asia: Tradition and Transformation. Boston, H oughton M ifflin, 1973

5. U ba L: Asian Americans: Personality Patterns, I dentity, and M ental $\mathrm{H}$ ealth. $\mathrm{New}$ York, Guilford, 1994

6. H su FLK: Americans and Chinese: Passage to Differences. H onolulu, U niversity Press of H awaii, 1981

7. Lin KM: Culture and DSM-IV: AsianAmerican perspectives, in Culture and Psychiatric Diagnosis. E dited by M ezzich JE, Kleinman $\mathrm{A}$, $\mathrm{F}$ abrega $\mathrm{H}$, et al. Washington, D C, American Psychiatric Press, 1994

8. Roland A: In Search of Self in India and Japan. Princeton, NJ, Princeton U niversity Press, 1988

9. Marsella AJ, D eVos G, H su FLK: Culture and Self: Asian and Western Perspectives. N ew York, Tavistock, 1985

10. Lin KM, Cheung FK, Smith M W, et al: The use of psychotropic medications in working with Asian patients, in Working With Asian Americans: A Guide for Clinicians. E dited by L ee E. N ew York, Guilford, 1997

11. Jew CC, Brody SA: M ental illness among the Chinese: hospitalization rates over the past century. Comprehensive Psychiatry 2: 129-134, 1967

12. Sue $S, M$ orishima J: The $M$ ental $H$ ealth of Asian-Americans. San Francisco, JosseyBass, 1982

13. Cheung FK: The mental health status of Asian Americans. Clinical Psychologist 34: 23-24, 1980

14. Bui KV, Takeuchi DT: E thnic minority adolescents and the use of community mental health care services. American Journal of Community Psychology 20:403-417, 1992

15. Lin TY: Psychiatry and Chinese culture. Western Journal of M edicine 139:862-867, 1983

16. Lin TY, Lin M C: Service delivery issues in Asian-North American communities. American Journal of Psychiatry 135:454456, 1978

17. Lin KM, Inui T, Kleinman A: Sociocultural determinants of help-seeking behavior of patients with mental illness. J ournal of Nervous and M ental D isease 170:78-85, 1982

18. Zatzick D F , L u F G: The ethnic/minority focus unit as a training site in transcultural psychiatry. Academic Psychiatry 15:218225, 1991

19. Kinzie JD, M anson S: Five years' experience with Indochinese refugee psychiatric patients. Journal of Operational Psychiatry 14:13-19, 1983

20. Cheung FK, Snowden LR: Community mental health and ethnic minority populations. Community $\mathrm{M}$ ental $\mathrm{H}$ ealth Journal 26:277-291, 1990

21. Yu E SH , Cypress BK : Visits to physicians by 
Asian/Pacific Americans. M edical Care 20: 809-820, 1982

22. M ezzich JE, F abrega H, Kleinman A: Cultural validity and DSM -IV. Journal of Nervous and M ental D isease 180:4, 1992

23. M ezzich JE, Kleinman $A, F$ abrega $H$, et al: Culture and Psychiatric Diagnosis. Washington, DC, American Psychiatric Press, 1995

24. F abrega $\mathrm{H}$ : Psychiatric diagnosis: a cultural perspective. Journal of $\mathrm{N}$ ervous and $\mathrm{M}$ ental D isease 175:383-394, 1987

25. Roukema R, Fadem BH, James $B$, et al: Bipolar disorder in a low socioeconomic population: difficulties in diagnosis. Journal of Nervous and Mental D isease 72:76-79, 1984

26. M ukherjee S, Shukla S, Woodle J, et al: M isdiagnosis of schizophrenia in bipolar patients: a multiethnic comparison. American Journal of Psychiatry 140:1571-1574, 1983

27. Chun CA, E nomoto $K$, Sue $S$ : $H$ ealth care issues among Asian Americans: implications of somatization, in $\mathrm{H}$ andbook of $\mathrm{D}$ iversity in Health Psychology. Edited by Kato PM, M ann T. New York, Plenum, 1996

28. F laherty JA, G aviria F, Pathak D, et al: Developing instruments for cross-cultural psychiatric research. Journal of Nervous and M ental D isease 176:257-263, 1988

29. Tseng WS, Asai M, Liu J Q, et al: M ulti-cultural study of minor psychiatric disorders in Asia: symptom manifestations. International Journal of Social Psychiatry 36:252-264, 1990

30. Kleinman A, Kleinman J: Somatization: the interconnections in Chinese society among culture, depressive experiences, and the meanings of pain, in Culture and Depression: Studies in the Anthropology and Cross-C ultural Psychiatry of Affect and D isorder. Edited by Kleinman A, Good BJ . Berkeley, University of California Press, 1985

31. Kleinman A: Rethinking Psychiatry. New York, F ree Press, 1988

32. Cheung F M , L au BW: Situational variations of help-seeking behavior among Chinese patients. Comprehensive Psychiatry 23: 252-262, 1982

33. Cheung FM : An overview of psychopathology in $\mathrm{H}$ ong Kong with special reference to somatic presentation, in Chinese Culture and $\mathrm{M}$ ental $\mathrm{H}$ ealth. E dited by Tseng WS, Wu D. Orlando, Fla, Academic, 1985

34. Lin KM, Tazuma L, Masuda M: Adaptational problems of Vietnamese refugees: part I. health and mental health status. Archives of G eneral Psychiatry 36:955-961, 1979

35. Lin KM, Masuda M, Tazuma L: Adaptational problems of Vietnamese refugees: part III. case studies in clinic and field adaptive and maladaptive. Psychiatric Journal of the University of Ottawa 7:173-183, 1982

36. M asuda M, Lin KM, Tazuma L: Adaptational problems of Vietnamese refugees: part II. life changes and perception of life events. Archives of General Psychiatry 37: 447-450, 1980

37. Cheung FM: F acts and myths about somatization among the Chinese, in Chinese Societies and $M$ ental $\mathrm{H}$ ealth. E dited by Lin TY, T seng WS, Yeh E K. H ong K ong, Oxford U niversity Press, 1995

38. F abrega $\mathrm{H}$ : The concept of somatization as a cultural and historical product of Western medicine. Psychosomatic M edicine 52:653672,1990

39. Kleinman A: What is specific to Western medicine? in Companion Encyclopedia of the $\mathrm{H}$ istory of M edicine. E dited by Bynum WF, Porter R. L ondon, Routledge, 1993

40. Lin KM: Traditional Chinese medical beliefs and their relevance for mental illness and psychiatry, in Normal and Abnormal Behavior in Chinese Culture. Edited by Kleinman A, L in TY. Boston, Reidel, 1980

41. H su L K G, F olstein M F : Somatoform disorders in Caucasian and Chinese Americans. Journal of Nervous and Mental Disease 185:382-387, 1997

42. Kleinman $A$ : Social Origins of $D$ istress and D isease: D epression, N eurasthenia, and Pain in Modern China. N ew $\mathrm{H}$ aven, Yale University Press, 1986

43. Young D S: Neurasthenia and related problems. Culture, M edicine, and Psychiatry 13: 131-138, 1989

44. Lin TY: N eurasthenia revisited: its place in modern psychiatry. Culture, M edicine, and Psychiatry 13:105-129, 1989

45. Kleinman A: N eurasthenia and depression: a study of somatization and culture in China. Culture, Medicine, and Psychiatry 6: 117-190, 1982

46. The ICD-10 Classification of Mental and Behavioural Disorders: Clinical Descriptions and Diagnostic Guidelines. Geneva, W orld H ealth Organization, 1992

47. Zheng YP, Lin KM, Takeuchi DT, et al: An epidemiological study of neurasthenia in Chinese Americans in Los Angeles. Comprehensive Psychiatry 38:249-259, 1997

48. Lin KM: Hwa-byung: a Korean culturebound syndrome? American Journal of Psychiatry 140:105-107, 1983

49. Lin KM, Lau JK, Yamamoto J, et al: H wabyung: a community study of Korean Americans. Journal of Nervous and M ental Disease 180:386-391, 1992

50. M in SK: A study of the concept of hwabyung. Journal of the Korean N europsychiatric Association 28:604-616, 1989

51. D iagnostic and Statistical M anual of M ental D isorders, 4th ed. Washington, D C, American Psychiatric Association, 1994

52. Kirmayer LJ, Weiss M G : Cultural considerations for somatoform disorders, in DSM IV Sourcebook. Edited by Widiger TA, F rances $A$ J, Pincus $H A$, et al. Washington, DC, American Psychiatric Press, 1997

53. Leslie C: Asian M edical Systems: A Comparative Study. Berkeley, U niversity of Cali- fornia Press, 1976

54. Kleinman A: Patients and Healers in the Context of Culture. Berkeley, University of California Press, 1980

55. E isenberg D M , Kessler RC, F oster C, et al: Unconventional medicine in the United States: prevalence, cost, and patterns of use. New England Journal of Medicine 328: 246-252, 1993

56. Smith M, Lin KM, M endoza R: "Non-biological" issues affecting psychopharmacotherapy: cultural considerations, Psychopharmacology and Psychobiology of Ethnicity. Edited by Lin KM, Poland R, N akasaki G. Washington, D C, American Psychiatric Press, 1993

57. Chan $C$, Chang J: The role of Chinese medicine in N ew York City's Chinatown. American Journal of Chinese M edicine 4:31-45, 1976

58. Lee E: Working With Asian Patients: A Guide for Clinicians. N ew York, Guilford, 1997

59. Falloon IRH: Family $M$ anagement of Schizophrenia. Baltimore, John Hopkins University Press, 1985

60. Lin KM, M iller M H, Poland RE, et al: $E$ thnicity and family involvement in the treatment of schizophrenic patients. Journal of Nervous and M ental D isease 179:631-633 1991

61. Acosta FX, Yamamoto J, E vans LA: E ffective Psychotherapy for Low-Income and M inority Patients. N ew York, Plenum, 1982

62. Kinzie JD : L essons from cross-cultural psychotherapy. American Journal of Psychotherapy 32:510-520, 1978

63. H su J, T seng WS: I ntercultural psychotherapy. Archives of General Psychiatry 27: 700-705, 1972

64. F rank JD : Persuasion and H ealing: A Comparative Study of Psychotherapy. N ew York, Schocken, 1974

65. Yi K: Psychoanalytic psychotherapy with Asian clients: transference and therapeutic considerations. Psychotherapy 32:308-315, 1995

66. Sue S, Zane N : The role of culture and cultural techniques in psychotherapy: a critique and reformulation. American Psychologist 42:37-45, 1987

67. Kopelowicz A, Liberman R P: Biobehavioral treatment and rehabilitation of schizophrenia. $\mathrm{H}$ arvard Review of Psychiatry 3:55-64, 1995

68. Lin KM, Poland RE, Nakasaki G: Psychopharmacology and Psychobiology of Ethnicity. Washington, DC, American Psychiatric Press, 1993

69. Lin KM, Poland RE, Wan $Y$, et al: The evolving science of clinical pharmacogenetics: clinical and ethnic perspectives. Psychopharmacology Bulletin 32:205-217, 1996

70. Lin KM : Psychopharmacology in cross-cultural psychiatry. Mount Sinai Journal of M edicine 63:283-284, 1996 